PROCEEDINGS OF THE

AMERICAN MATHEMATICAL SOCIETY

Volume 131, Number 12, Pages 3919-3923

S 0002-9939(03)07002-3

Article electronically published on May 28, 2003

\title{
A PROOF OF NOGURA'S CONJECTURE
}

\author{
STEVO TODORCEVIC \\ (Communicated by Alan Dow)
}

\begin{abstract}
Answering a question of T. Nogura (1985), we show using the Open Coloring Axiom that the weak diagonal sequence property is preserved by taking products whenever the products themselves are Fréchet. As an application we show, using the same assumption, that the product of two Fréchet groups is Fréchet provided it is sequential. Recall that the product of two Fréchet groups may not be sequential.
\end{abstract}

Recall that a given topological space $X$ (implicitly assumed to be at least Hausdorff) is sequential if every nonclosed subset $A$ of $X$ contains a sequence which converges to a point outside of $A$. Recall also that a space $X$ is said to be Fréchet if every subset $A$ of $X$ which accumulates to some point $x$ in $X$ contains a sequence which converges to $x$. It is usually in the realm of Fréchet spaces that one considers various ways to obtain a converging sequence out of a given sequence of converging sequences (see, e.g., 1], [2], [3], 4], [5]). For example, the well-known diagonalsequence property states that if $\left\{x_{n k}\right\}$ is a double-indexed sequence of members of $X$ such that for some $x \in X$ and all $n, x_{n k} \rightarrow_{k} x$, then for each $n$ we can choose $k(n)$ such that

$$
x_{n k(n)} \rightarrow{ }_{n} x .
$$

This turns out to be quite a strong property preserved under products and imposing first-countability when $X$ is countable and the topology of $X$ is an analytic subset of $2^{X}$ (see [10]). Consider however the weak diagonal-sequence property which asserts that we can make the choice $k(n)$ in such a way that some infinite subsequence of $\left\{x_{n k(n)}\right\}$ converges to $x$ rather than the sequence itself. This property seems to be considerably less restrictive than the diagonal sequence property as it can be seen, for example, from a result of Nyikos [3] which states that every Fréchet topological group has the weak diagonal sequence property. It turns out that in general the weak diagonal sequence property is not a productive property which motivated T. Nogura [2] to ask if the property is productive under some restriction such as the following.

Question 1 ([2, 3.15]). Suppose $X$ and $Y$ are two Fréchet spaces with the weak diagonal sequence property. Suppose further that their product $X \times Y$ is Fréchet. Does $X \times Y$ have the weak diagonal sequence property?

Received by the editors August 1, 2002 and, in revised form, August 6, 2002.

2000 Mathematics Subject Classification. Primary 03E65, 54B10, 54D55.

Key words and phrases. Fréchet space, diagonal sequence property.

(C)2003 American Mathematical Society 
The purpose of this note is to give a positive answer to Nogura's question assuming the Open Coloring Axiom [8, §8]. The use of OCA is quite natural here, as it is easily seen (see [6]) that the existence of a nontrivial coherent sequence of functions $c_{f}: \Gamma_{f} \rightarrow 2\left(f \in \mathbb{N}^{\mathbb{N}}\right)$ gives the negative answer, where for $\left(f \in \mathbb{N}^{\mathbb{N}}\right)$ we put

$$
\Gamma_{f}=\left\{(n, k) \in \mathbb{N}^{2}: k \leq f(n)\right\} .
$$

In fact, the recent article of Simon and Tironi [7], which motivated our work, shows that the existence of some strong counterexample to Nogura's conjecture is actually equivalent to the existence of a nontrivial coherent sequence $c_{f}: \Gamma_{f} \rightarrow 2\left(f \in \mathbb{N}^{\mathbb{N}}\right)$. Recall that OCA implies that every coherent sequence $c_{f}: \Gamma_{f} \rightarrow 2\left(f \in \mathbb{N}^{\mathbb{N}}\right)$ is trivial i.e., induced modulo-finite by a single map $d: \mathbb{N}^{2} \rightarrow 2$ (see $[8, \S 8]$ ). What we show here is that a different application of OCA gives us a proof of Nogura's conjecture in its full generality.

Theorem 2 (OCA). Suppose that $X$ and $Y$ are Fréchet spaces with the weak diagonal sequence property. Suppose further that their product $X \times Y$ is Fréchet. Then $X \times Y$ has the weak diagonal sequence property.

Proof. Suppose there is a pair of spaces $X$ and $Y$ satisfying the hypothesis of the theorem but their product $X \times Y$ fails to have the weak diagonal sequence property. A Fréchet space without the weak diagonal sequence property is easily seen to contain a copy of the sequential fan $S_{\omega}$, the topological space on $\mathbb{N}^{2} \cup\{\infty\}$ with only $\infty$ nonisolated while the neighborhood base of $\infty$ is generated by the complements of the sets of the forms $\Gamma_{f}\left(f \in \mathbb{N}^{\mathbb{N}}\right)$. So, from our assumption, one easily constructs two Fréchet topologies $\tau_{X}$ and $\tau_{Y}$ on $\mathbb{N}^{2} \cup\{\infty\}$ with $\infty$ as the only nonisolated point such that $\tau_{X}$ and $\tau_{Y}$ both have the weak diagonal sequence property while the topology of $S_{\omega}$ is generated by $\tau_{X} \cup \tau_{Y}$ as a subbasis. For $n \in \mathbb{N}$, set

$$
C_{n}=\{n\} \times \mathbb{N} .
$$

Note that each $C_{n}$ is a converging sequence in both topologies $\tau_{X}$ and $\tau_{Y}$. Let

$$
\begin{aligned}
& \mathcal{A}=\left\{A \subseteq \mathbb{N}^{2}: A \rightarrow_{\tau_{X}} \infty \text { and } A \cap C_{n} \text { is finite for all } n\right\}, \\
& \mathcal{B}=\left\{B \subseteq \mathbb{N}^{2}: B \rightarrow_{\tau_{Y}} \infty \text { and } B \cap C_{n} \text { is finite for all } n\right\} .
\end{aligned}
$$

Thus, $\mathcal{A}$ (respectively, $\mathcal{B}$ ) is the family of all sequences that converge to $\infty$ relative to $\tau_{X}$ (respectively, relative to $\tau_{Y}$ ) and which are orthogonal to each member of the sequence $\left\{C_{n}\right\}$ of converging sequences. Let

$$
\mathcal{X}=\{(A, B) \in \mathcal{A} \times \mathcal{B}: A \cap B=\emptyset\} .
$$

We endow $\mathcal{X}$ with the standard separable metric topology induced from $2^{\mathbb{N}^{2}}$. Consider the following subset of the set $\mathcal{X}^{[2]}$ of all unordered pairs of elements of $\mathcal{X}$ :

$$
\mathcal{K}=\left\{\left\{(A, B),\left(A^{\prime}, B^{\prime}\right)\right\} \in \mathcal{X}^{[2]}:\left(A \cap B^{\prime}\right) \cup\left(A^{\prime} \cap B\right) \neq \emptyset\right\} .
$$

Note that $\mathcal{K}$ is an open subset of $\mathcal{X}^{[2]}$, so OCA applies to it. Therefore, to finish the proof, it suffices to show that neither of the following two alternatives given by OCA is possible.

Case 1. There is uncountable $\mathcal{Y} \subseteq \mathcal{X}$ such that $\mathcal{Y}^{[2]} \subseteq \mathcal{K}$. We may assume that $\mathcal{Y}$ actually has size $\aleph_{1}$ and since under OCA subsets of $\mathbb{N}^{\mathbb{N}}$ of size $\aleph_{1}$ are bounded 
in the ordering of eventual dominance (see [8 $\S 8]$ ), going to a subset of $\mathcal{Y}$, we may assume that there is $f \in \mathbb{N}^{\mathbb{N}}$ such that

$$
A \cup B \subseteq \Gamma_{f} \text { for all }(A, B) \in \mathcal{Y} .
$$

Since $\Gamma_{f}$ does not accumulate to $\infty$ in $S_{\omega}$ and since $\tau_{X} \cup \tau_{Y}$ is a subbasis of the sequential fan $S_{\omega}$, we can find $U \in \tau_{X}$ and $V \in \tau_{Y}$ such that $\infty \in U \cap V$ and

$$
U \cap V \cap \Gamma_{f}=\emptyset \text {. }
$$

For $D \subseteq \mathbb{N}^{2}$ and $n \in \mathbb{N}$, set

$$
D / n=D \backslash(n \times \mathbb{N}) \text { and } D\lceil n=D \cap(n \times \mathbb{N}) .
$$

Note that for every $(A, B) \in \mathcal{Y}$ there is $n$ such that $A / n \subseteq U$ and $B / n \subseteq V$. So we can find $n \in \mathbb{N}$ and an uncountable subset $\mathcal{Y}_{0}$ of $\mathcal{Y}$ such that

(a) $A / n \subseteq U$ and $B / n \subseteq V$ for all $(A, B) \in \mathcal{Y}_{0}$,

(b) $A\left\lceil n=A^{\prime}\left\lceil n\right.\right.$ and $B\left\lceil n=B^{\prime}\left\lceil n\right.\right.$ for all $(A, B),\left(A^{\prime}, B^{\prime}\right) \in \mathcal{Y}_{0}$.

Pick two distinct elements $(A, B)$ and $\left(A^{\prime}, B^{\prime}\right)$ of $\mathcal{Y}_{0}$. Then the unordered pair $\left\{(A, B),\left(A^{\prime}, B^{\prime}\right)\right\}$ belongs to $\mathcal{K}$, and therefore,

$$
U \cap V \cap \Gamma_{f} \supseteq\left(A \cap B^{\prime}\right) \cup\left(A^{\prime} \cap B\right) \neq \emptyset,
$$

a contradiction.

Case 2. There is a decomposition

$$
\mathcal{X}=\bigcup_{n=0}^{\infty} \mathcal{X}_{n}
$$

such that $\left(\mathcal{X}_{n}\right)^{[2]} \cap \mathcal{K}=\emptyset$ for all $n$.

For $n \in \mathbb{N}$, set

$$
D_{n}=\bigcup\left\{A:(A, B) \in \mathcal{X}_{n} \text { for some } B\right\} .
$$

Then for every $(A, B) \in \mathcal{A} \times \mathcal{B}$ there is $n$ such that $(A \backslash B, B) \in \mathcal{X}_{n}$ and, therefore,

$$
A \subseteq^{*} D_{n} \text { and } B \cap D_{n}=\emptyset .
$$

Pick an ultrafilter $\mathcal{U}$ on $\mathbb{N}^{2}$ such that for every $S \in \mathcal{U}$ there are infinitely many $n$ such that $C_{n} \cap S$ is infinite. Let $\epsilon: \mathbb{N} \rightarrow 2$ be such that

$$
D_{n}^{\epsilon(n)} \in \mathcal{U} \text { for all } n \in \mathbb{N} \text {. }
$$

(Here $D_{n}^{1}=D_{n}$ and $D_{n}^{0}=\mathbb{N}^{2} \backslash D_{n}$.) Let $\left\{n_{k}\right\}_{k=0}^{\infty}$ be the strictly increasing sequence of elements of $\mathbb{N}$ such that $n_{k}$ is the minimal integer $>n_{k-1}$ with the property that

$$
E_{n_{k}}=C_{n_{k}} \cap \bigcap_{i=0}^{n_{k-1}} D_{i}^{\epsilon(i)}
$$

is infinite; $n_{-1}=0$. Applying the fact that $\tau_{X}$ has the weak diagonal sequence property to the sequence $\left\{E_{n_{k}}\right\}_{k=0}^{\infty}$ of $\tau_{X}$-converging sequences to $\infty$, we find $A \in \mathcal{A}$ such that

$$
A \subseteq \bigcup_{k=0}^{\infty} E_{n_{k}}
$$


and such that $A \cap E_{n_{k}}$ has at most one point for all $k$. Applying the fact that $\tau_{Y}$ has the weak diagonal sequence property to the same sequence $\left\{E_{n_{k}}\right\}_{k=0}^{\infty}$ of $\tau_{Y}$-converging sequences to $\infty$ we find $B \in \mathcal{B}$ such that

$$
B \subseteq \bigcup_{k=0}^{\infty} E_{n_{k}}
$$

and such that $B \cap E_{n_{k}}$ has at most one point for all $k$. Let $n$ be such that $(A \backslash B, B) \in$ $\mathcal{X}_{n}$, or in other words

$$
A \subseteq^{*} D_{n} \text { and } B \cap D_{n}=\emptyset .
$$

Pick $j$ such that $n_{j-1} \geq n$. Then

$$
A \cup B \subseteq^{*} \bigcup_{k=j}^{\infty} E_{n_{k}} \subseteq D_{n}^{\epsilon(n)},
$$

a contradiction. This finishes the proof of Theorem 2 .

Remark 3. If one assumes that in the hypothesis of Theorem 2 the spaces $X$ and $Y$ are countable and their topologies $\tau_{X}$ and $\tau_{Y}$ are analytic subsets of $2^{X}$ and $2^{Y}$, respectively, then the conclusion of Theorem 2 is true with no extra set-theoretic assumption. This follows from a characterization of bisequentiality of analytic topologies given in [11]:

Theorem 4. An analytic topological space is bisequential if and only if it is Fréchet and has the weak diagonal sequence property.

Recall that a topological space $X$ is bisequential if it has the property that if an ultrafilter $\mathcal{U}$ on $X$ converges to a point $x$ in $X$, then $\mathcal{U}$ contains a sequence of sets that converge to the point $x$. This is yet another productive convergence property which strengthens the Fréchet property as well as the weak diagonal sequence property (see, e.g., [4).

Recall now that the product $G \times H$ of two Fréchet groups $G$ and $H$ may fail to be Fréchet (see [9]). In fact the product $G \times H$ given in [9] is not even countably tight. However, finding such a product $G \times H$ which is countably tight or sequential but not Fréchet is a subtle matter. For example, finding such a product would solve yet another well-known open problem in the area (due to V.I. Malyhin) which asks whether Fréchet property implies metrizability in the realm of countable topological groups (see [4]). While OCA is not sufficient for solving Malyhin's problem it might be relevant to the problem about products of Fréchet groups:

Problem 5. Find an assumption under which it is true that the product of two Fréchet groups is Fréchet if and only if it is countably tight.

The following corollary of the proof of Theorem 2 shows that sequential product or two Fréchet groups is always Fréchet, thus answering Question 6.13(i) of [4].

Theorem 6 (OCA). The product of two Fréchet groups is Fréchet if and only if it is sequential.

Proof. Clearly, we may assume that the given two Fréchet groups $G$ and $H$ are countable. Suppose that the product group $K=G \times H$ is sequential but not Fréchet. It is well known and easily seen that every countable sequential space which is not Fréchet contains a closed copy of the Arens space [1], the topological space on $\mathbb{N}^{2} \cup\{\infty\}$ with $\infty$ and $(n, 0)(n \in \mathbb{N})$ as nonisolated points, the neighborhood 
base of $\infty$ is generated by the complements of the sets of the forms $\Gamma_{f}\left(f \in \mathbb{N}^{\mathbb{N}}\right)$ and $\{n\} \times \mathbb{N}(n \in \mathbb{N})$, and the neighborhood base of a point of the form $(n, 0)$ is generated by tails of the sequence $(n, k)(k \in \mathbb{N})$. Note that if $\left\{x_{n k}\right\}$ is a doubleindexed sequence which together with $e_{K}$ forms a (natural) closed copy of the Arens space in $K$, then $\left\{x_{n k} x_{n 0}^{-1}\right\}$ together with $e_{K}$ forms a (natural) closed copy of the sequential fan $S_{\omega}$ in $K$. We have already mentioned the result of Nyikos [3] that Fréchet groups have the weak diagonal sequence property. Now observe that the proof of Theorem 2 shows that the product of two countable Fréchet spaces with the weak diagonal sequence property does not contain a closed copy of $S_{\omega}$. This finishes the proof.

Remark 7. (i) According to a result of [5] the use of an additional axiom to prove Theorem 6 is in some sense necessary.

(ii) Again if one assumes that in the hypothesis of Theorem 6 the groups $G$ and $H$ are countable and their topologies $\tau_{G}$ and $\tau_{H}$ are analytic subsets of $2^{G}$ and $2^{H}$, respectively, then the conclusion of Theorem 6 is true with no extra set-theoretic assumption. This follows from the following metrization theorem from [11] which gives the positive answer to the effective version of Malyhin's problem:

Theorem 8. A countable Fréchet topological group $G$ is metrizable if and only if its topology $\tau_{G}$ is an analytic subset of $2^{G}$.

\section{REFERENCES}

[1] R. Arens, Note on convergence in topology, Math. Magazine 23 (1950), 229-234. MR 12:271h

[2] T. Nogura, The product of $\left\langle\alpha_{i}\right\rangle$-spaces, Topology and its Appl. 21(1985), 251-259. MR 87d:54006

[3] P. Nyikos, Metrizability and the Fréchet-Urysohn property in topological groups, Proc. Amer. Math. Soc. 83 (1981), 793-801. MR 82k:54049

[4] D. Shakhmatov, Convergence in the presence of algebraic structure, in: Recent Progress in Topology (M. Husek and J. van Mill, eds.), to appear.

[5] A. Shibakov, Examples of sequential topological groups under the continuum hypothesis, Fund. Math. 151 (1996), 107-120. MR 97k:54021

[6] P. Simon, A hedgehog in a product, Acta Univ. Carolinae Math. Phys. 39 (1998), 147-153. MR 2000d:54033

[7] P. Simon and G. Tironi, No hedgehog in the product?, Comment. Math. Univ. Carolinae 43 (2002), 349-361.

[8] S. Todorcevic, Partition Problems in Topology, Contemp. Math., Vol. 84, American Math. Soc., Providence, RI, 1989. MR 90d:04001

[9] S. Todorcevic, Some applications of $S$ and L combinatorics, Ann. New York Acad. Sci. 705(1993), 130-167. MR 95j:54006

[10] S. Todorcevic, Analytic Gaps, Fund. Math. 150(1996), 55-66. MR 98j:03070

[11] S. Todorcevic and C. Uzcategui Analytic k-spaces, preprint, 2000.

Université Paris 7 - C.N.R.S., UMR 7056, 2, Place Jussieu, 75251 Paris Cedex 05, FRANCE

E-mail address: stevo@math.jussieu.fr 